\title{
Supplement to Limiting Spectral Distribution of Sample Autocovariance Matrices
}

\author{
ANIRBAN BASAK*1 ${ }^{* 1}$ ARUP BOSE ${ }^{\dagger 2}$ and SANCHAYAN SEN ${ }^{\ddagger 3}$ \\ ${ }^{1}$ Department of Statistics, Stanford University, USA. E-mail: anirbanb@stanford.edu \\ ${ }^{2}$ Statistics and Mathematics Unit, Indian Statistical Institute, INDIA. \\ E-mail: bosearu@gmail.com \\ ${ }^{3}$ Courant Institute of Mathematical Sciences, USA. E-mail: sen@cims.nyu.edu
}

This is a supplementary file to our main article Limiting Spectral Distribution of Sample Autocovariance Matrices

\section{Simulations}

Recall that none of the LSDs have a nice description. Following the suggestion of one of the Referees, we present a few simulations.

Figure 1 shows the simulation results for $\Gamma_{n}(X)$, and $\Gamma_{n}^{*}(X)$, with different distributions for $\left\{\varepsilon_{t}\right\}$. They confirm that the limits are indeed universal. It may be noted that the ESD (and hence the LSD) of $\Gamma_{n}^{*}(X)$ has some mass on the negative axis.

Figure 2 shows how the LSD of Type I banded $\Gamma_{n}(X)$ changes with the model as well as the value of the parameter $\alpha$ (note that for $\alpha=1$ Type I banded $\Gamma_{n}(X)$ is the original $\Gamma_{n}$ matrix). The unbounded nature of the LSD is evident from these simulations. The third row of the figure with $\alpha=m / n=0.01 \approx 0$ demonstrates that the LSD of $\Sigma_{n}(X)$ and $\Gamma_{n}(X)$ are virtually indistinguishable for $n=1000$, confirming that thinly banded ACVM are consistent for $\Sigma_{n}(X)$.

Figure 3 shows how the LSD varies as we change the bandwidth parameter $\alpha$ in Type I banded matrices. We consider two models in this figure: $\mathrm{AR}(1)$, and MA(1), both with $\theta=1 / 2$. Note that as $\alpha$ increases the right tail thickens, and the probability of being near zero decreases. Note that for $\alpha=0.05$ in the $\operatorname{AR}(1)$ model, and for $\alpha=0.06$ in the MA(1) model, there is considerable amount of mass in the negative axis. This mass reduces as the value of $\alpha$ decreases in both the models.

Figure 4 shows how the LSD of $\Gamma_{n}(X)$ varies as the parameter of the models change. As before we consider two models: $\operatorname{AR}(1)$, and MA(1). For the AR(1) model, as $\theta$ increases from 0 , we note that the tail thickens, and the mass near zero decreases as before. Same phenomenon is observed for the MA(1) model. Note that $\theta=0$ corresponds to the case

\footnotetext{
* Supported by Melvin and Joan Lane endowed Stanford Graduate Fellowship fund.

${ }^{\dagger}$ Research supported by J.C.Bose Fellowship, Govt. of India.

‡Supported by NYU graduate fellowship under Henry M. MacCracken Program.
} 
where $\left\{X_{t}\right\}$ are i.i.d. For the $\operatorname{AR}(1)$ model, when $\theta$ approaches 1 , i.e. when the process is near non-stationary the LSD becomes very flat, and its tail becomes huge. This is evident from the figure for $\theta=0.9$.
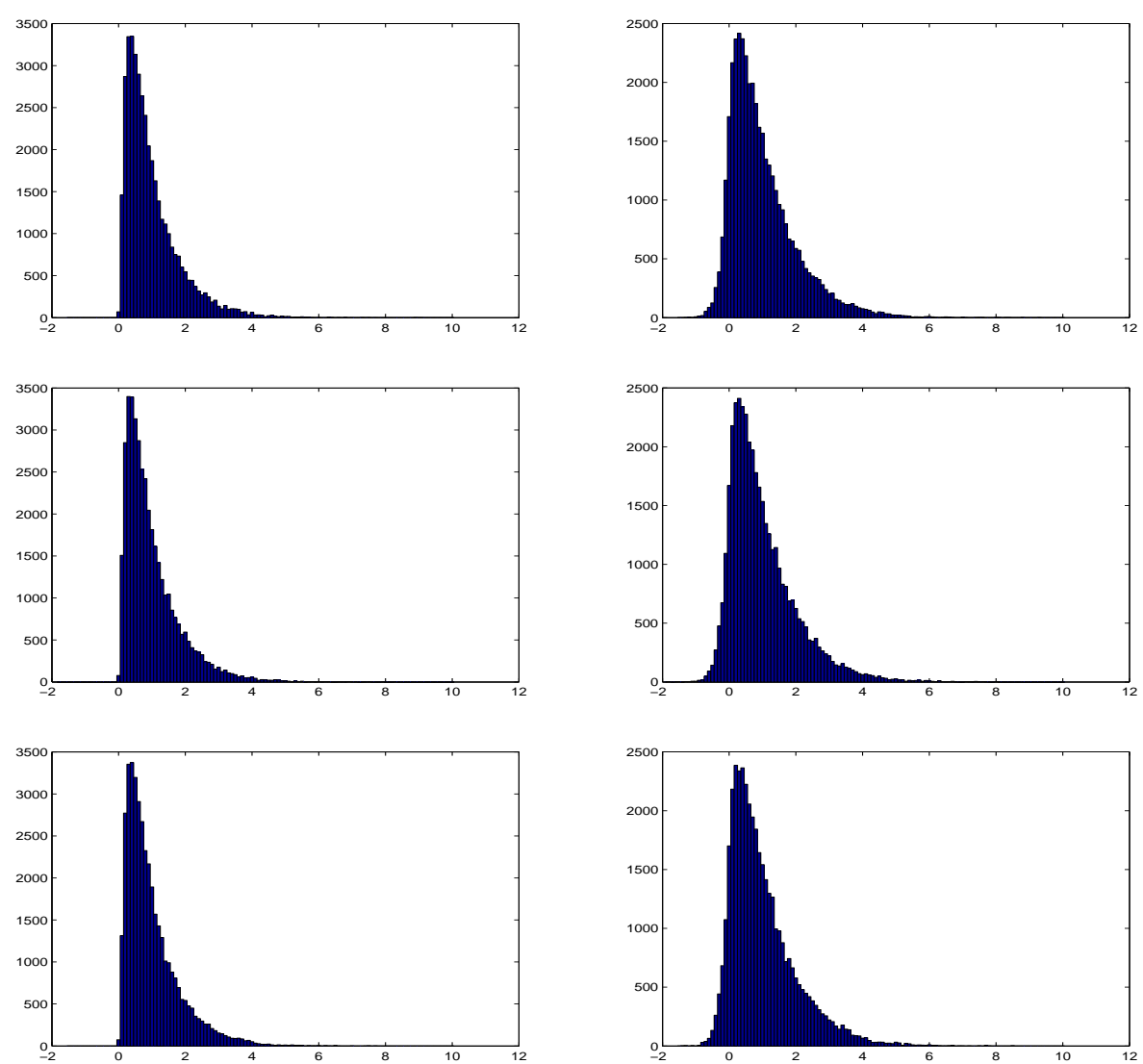

Figure 1. Histograms of the empirical distribution of eigenvalues of 100 realizations of $\Gamma_{n}(X)$ (left column) and $\Gamma_{n}^{*}(X)$ (right column), with $n=400$, when $X_{t}$ is i.i.d. Normal $(0,1)$ (top row), standardized exponential(1) (middle row) and standardized Bernoulli(0.5) (bottom row). 

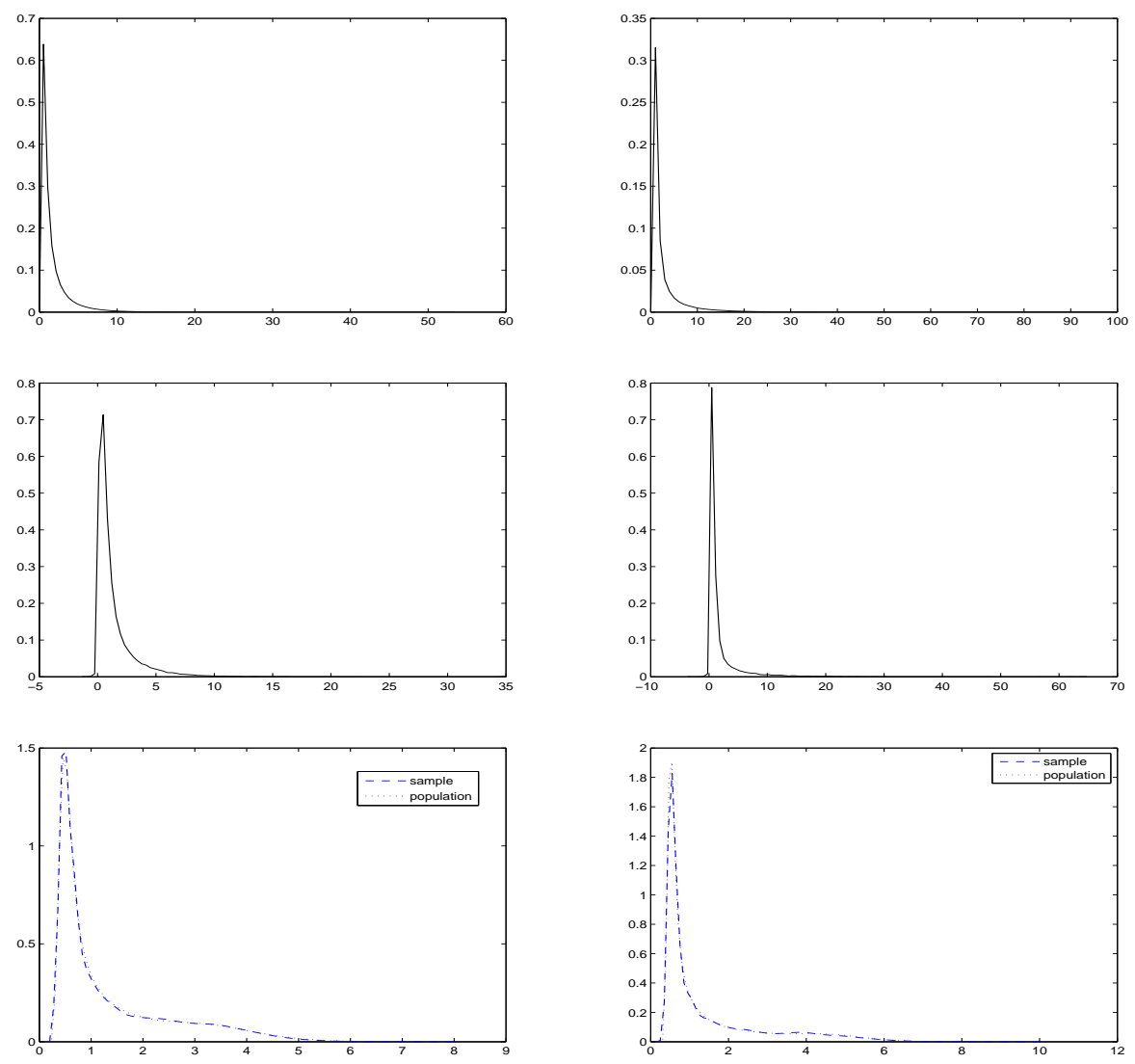

Figure 2. Kernel density estimates of the ESD 100 realizations of: Type I banded $\Gamma_{n}(X)$ with $n=1000$; $X \sim A R(1), \varepsilon_{t} \sim N(0,1), \theta=1 / 2$ (left column) and $X \sim A R(2), \varepsilon_{t} \sim N(0,1), \theta_{1}=1 / 2, \theta_{2}=1 / 16$ (right column) (i) $\alpha=1$ (top row) (ii) $\alpha=1 / 2$ (middle row) and (ii) $\alpha \approx 0$ ( $m=10$ ) (bottom row). Note that the case $\alpha=1$ corresponds to the original $\Gamma_{n}(X)$ wthout any banding structure. In the last row the density of the LSD of $\Sigma_{n}(X)$ has been superimposed and is virtually indistinguishable from the ESD of $\Gamma_{n}(X)$. 

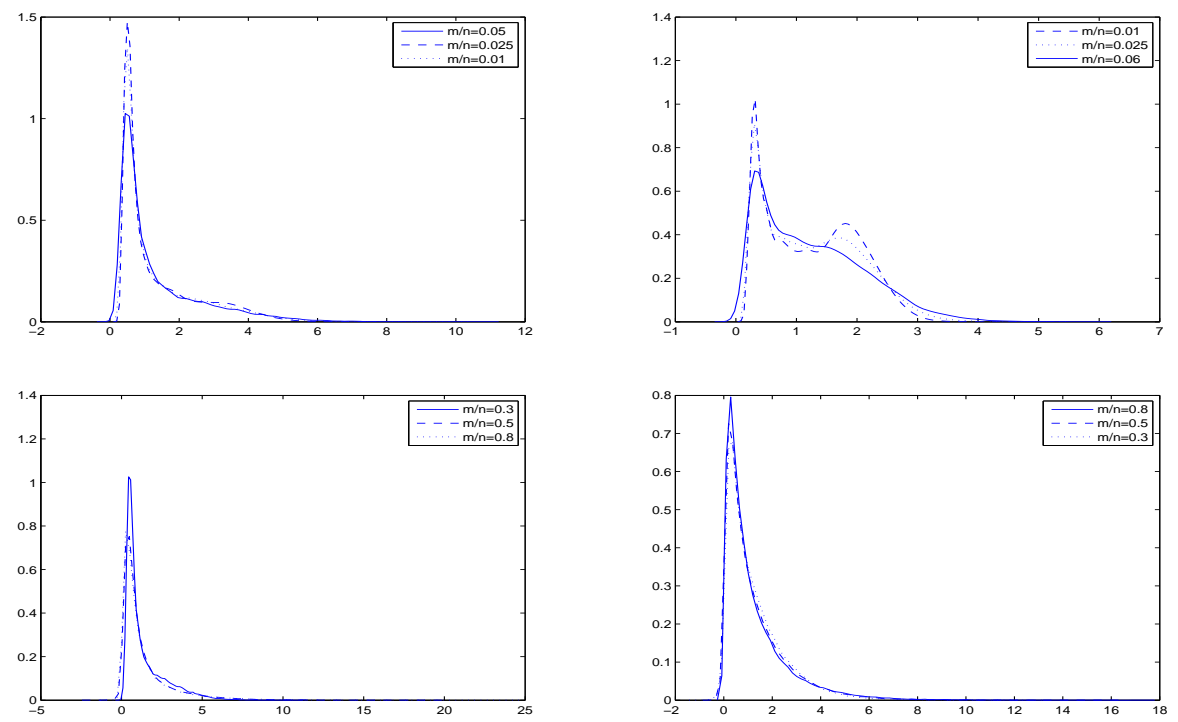

Figure 3. Kernel density estimates of the ESD 100 realizations of: Type I banded $\Gamma_{n}(X)$ with $n=1000$; $X \sim A R(1), \varepsilon_{t} \sim N(0,1), \theta=1 / 2$ (left column) and $X \sim M A(1), \varepsilon_{t} \sim N(0,1), \theta_{1}=1 / 2$, (right column) (i) $\alpha=0.01,0.25,0.5$ (top row) for AR process, and $\alpha=0.01,0.25,0.6$ for the MA process, and (ii) $\alpha=0.3,0.5,0.8$ (bottom row) for both the AR and MA processes.
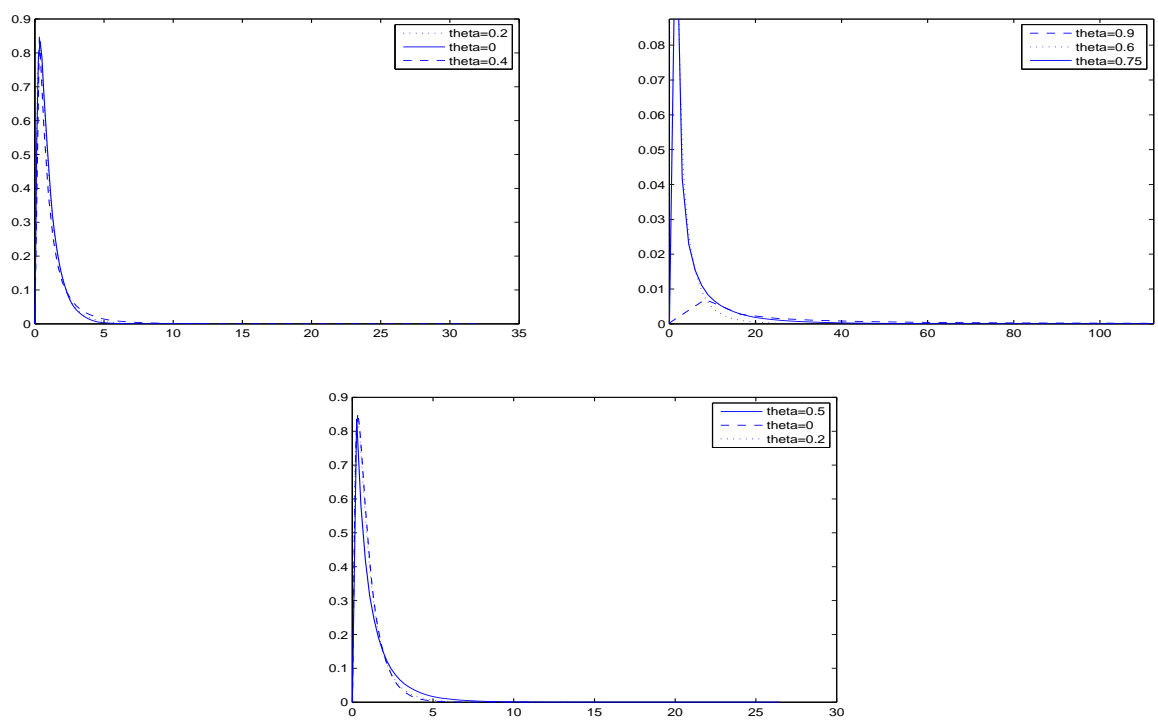

Figure 4. Kernel density estimates of the ESD 100 realizations of: $\Gamma_{n}(X)$ with $n=1000 ; X \sim A R(1)$, $\varepsilon_{t} \sim N(0,1)$, (top row), (i) $\theta_{1}=0,0.2,0.4$ (left column), and (ii) $\theta_{1}=0.6,0.75,0.9$ (right column), and $X \sim M A(1), \varepsilon_{t} \sim N(0,1), \theta_{1}=0,0.2,0.5$, (bottom row). 